\title{
An association of adverse psychosocial factors with diabetes mellitus: a meta-analytic review of longitudinal cohort studies
}

\author{
Y. Chida • M. Hamer
}

Received: 3 June 2008 / Accepted: 11 August 2008 / Published online: 20 September 2008

(C) Springer-Verlag 2008

\begin{abstract}
Aims/hypothesis There has been substantial interest in the association between psychosocial stress and risk of diabetes mellitus, but no data on the systematic quantification of the causal relationship have been published. This analysis aims to evaluate the association between adverse psychosocial factors and diabetes mellitus.

Methods We performed a search of Medline, PsycINFO, Web of Science and PubMed up to July 2008. The studies included were prospective cohort studies investigating the association between adverse psychosocial factors and risk of diabetes mellitus.

Results There were 22 relationships between psychosocial factors and disease-related factors (in 14 papers), of which 16 evaluated the associations of adverse psychosocial factors with diabetes control in diabetic populations and six evaluated the associations of adverse psychosocial factors with the incidence of diabetes in populations without any diagnosed diabetes. The overall meta-analysis demonstrated that adverse psychosocial factors were significantly associated with poor diabetes control (combined correlation coefficient, $r=0.096, p=0.006$ ), whereas adverse psychosocial factors were not associated with incident diabetes mellitus. More notably, sensitivity analyses showed that low social support was more robustly associated with poor diabetes control than stressful events
\end{abstract}

Electronic supplementary material The online version of this article (doi:10.1007/s00125-008-1154-1) contains supplementary material, which is available to authorised users.

Y. Chida $(\square) \cdot$ M. Hamer

Psychobiology Group, Department of Epidemiology and

Public Health, University College London,

1-19 Torrington Place,

London WC1E 6BT, UK

e-mail: y.chida@ucl.ac.uk per se or stress-prone personality or coping style, and that adverse psychosocial factors were associated with poor control of type 1 and type 2 diabetes.

Conclusions/interpretation The current review revealed a detrimental association of psychosocial factors with the prognosis of both type 1 and type 2 diabetes. However, any aetiological effect of adverse psychosocial factors remains elusive as a result of the small number of individuals enrolled in the cohorts studied.

Keywords Meta-analysis · Mind and body connection . Psychosocial stress $\cdot$ Psychoneuroendocrinology

\section{Abbreviation \\ SES socioeconomic status}

\section{Introduction}

As early as the 17th century, Thomas Willis, the first physician to write the English medical treatise about diabetes mellitus, mentioned that 'nervous juice hurtful to other humors and prolonged sorrow appeared to be important risk factors for diabetes mellitus' [1]. Over the past 20 years, a body of studies has investigated associations between adverse psychosocial factors and diabetes mellitus development and prognosis. Exposure to psychosocial stress is associated with a number of pathophysiological mechanisms that make an association between stress and diabetes risk theoretically plausible. For example, psychosocial stress can stimulate the hypothalamus-pituitary-adrenal axis, the sympathetic nervous system and inflammatory pathways known to affect glucose metabolism $[2,3]$. However, evidence from clinical trials on the 
efficacy of psychological interventions for the treatment of diabetes remains equivocal $[4,5]$. The several reviews on this topic have reported disparate findings. This may be because some reviews have not distinguished between prospective studies and cross-sectional or retrospective case-control studies [6-8]. Cross-sectional and retrospective case-control studies are subject to recall bias caused by diabetes mellitus diagnosis or memory distortion, and cannot conclusively detect a longitudinal association between predictors and outcome variables. Furthermore, none of the previous reviews have used meta-analytic techniques to quantify the extent to which adverse psychosocial factors affect diabetes mellitus [6-10].

Psychosocial stress can be considered a product of exposure to a stressor and the human response to it. Thus, several factors are relevant to stress responses, including cognitive appraisals, behavioural coping and the use of social support [3, 11]. The conceptualisation of social support covers instrumental support, including both financial and assistance with tasks, emotional/appraisal support, information, companionship and self-esteem support [12]. Accordingly, several psychosocial categories have been defined: (1) stressful events (e.g. life events, job stress, severe chronic stress and daily stress); (2) stress-prone personality or coping style (e.g. avoidant coping, denial coping, neuroticism, hopelessness); and (3) poor social support (e.g. poor social participation, poor stable partnership, poor family contact, loneliness).

The aim of this article is to conduct a systematic review and meta-analysis of prospective cohort studies to explore and quantify the putative causal associations of adverse psychosocial factors with the development and prognosis of diabetes mellitus. The following questions will be addressed: (1) What adverse psychosocial factors are associated with diabetes mellitus? (2) Do associations with adverse psychosocial factors differ according to methodological study quality, follow-up periods and participant characteristics (age and type 1 or type 2 diabetes)? The roles of behavioural and biological pathways in the association between adverse psychosocial factors and diabetes mellitus are also discussed. Given that the disease burden of diabetes can in itself be considered a powerful chronic stressor, we hypothesised that the association between adverse psychosocial factors and diabetes prognosis would be stronger than the association with diabetes development.

\section{Methods}

Data sources and searches We developed a protocol using a widely recommended method for conducting systematic reviews of observational studies [13]. We searched the general bibliographic databases Medline (1966-July 2008), PsycINFO (1872-July 2008), Web of Science (1900-July 2008) and PubMed (1950-July 2008), and scrutinised reference lists from relevant reviews and articles. The main search strategy was diabetes mellitus AND (psychological stress OR psychosocial stress OR work stress OR life event OR life stress OR chronic stress OR social support OR personality OR coping) AND (prospective OR longitudinal).

Study selection We limited the current systematic review and meta-analysis to prospective studies. Cross-sectional and retrospective case-control studies are subject to recall bias and cannot conclusively identify the temporal association between predictors and outcome variables. As mentioned in the introduction, adverse psychosocial factors were divided into the following categories: (1) stressful events; (2) stress-prone personality or coping style; and (3) poor social support. Criteria for inclusion were as follows: (1) English language full-length publication in a peerreviewed journal; (2) prospective cohort design; (3) investigation of a longitudinal association of adverse psychosocial factors with diabetes mellitus control or incidence. If more than one kind of adverse psychosocial factor was assessed in one paper, the samples were included separately. If more than one type of diabetes mellitus control was assessed in one paper, the data for the longer term indicator of glycaemic control were included (e.g. $\mathrm{HbA}_{1 \mathrm{c}}$ rather than average selfmonitoring blood glucose levels for a week). Studies that used low socioeconomic status (SES) as an indicator for adverse psychosocial factors were excluded, because almost all studies included SES as a covariate, and low SES alone has many consequences other than greater stress experience [14, 15]. Studies that examined the association between diabetes and psychiatric illnesses or psychological distress, such as depression and anxiety, were excluded because there may be illness-related disturbances of physiological function in these patients [16]. If a cohort was analysed in more than one publication, the paper with shorter follow-up, smaller sample size or poorer study quality was excluded.

Data extraction and quality assessment A manual was prepared for coding the studies. The manual was revised during the coding to incorporate important aspects of the identified studies. The final list of variables included first author and publication year, cohort size with participant characteristics (country), follow-up duration (years), type of adverse psychosocial factors (method of measurement), controlled covariates, diabetes outcome (method of measurement), quality score and brief results/effect size (correlation coefficient $[r]$ in diabetes control, HR or RR with $95 \%$ CIs in diabetes incidence). The selected studies were categorised into two groups: associations between adverse psychosocial factors and diabetes control in 
diabetic patients; and associations between adverse psychosocial factors and diabetes incidence in a population without diabetes at enrolment. Categorising the studies in this manner enabled us to examine both the prognostic and aetiological effects of adverse psychosocial factors on diabetes. When primary sources provided insufficient data, we obtained additional information by other means, such as communication with the author or from indirect calculations.

We assessed all manuscripts for quality, since this can contribute to the potential bias associated with the effect estimation. In line with previous studies [17-19], we considered a study to be of good quality if (1) participants were recruited consecutively or randomly or a representative population was used; (2) explanatory variables were ascertained by validated instruments or clinical examination; (3) outcome variables were ascertained by validated instruments or clinical examination; and (4) possible covariates were controlled for, including age, sex, smoking, alcohol consumption, BMI or physical activity level, and SES, and for populations with diabetes mellitus, basal disease status and medical treatment. These quality criteria were scored one point each. Studies were placed into high or low quality categories according to whether or not they fulfilled three or more of these criteria.

Study inclusion and data extractions were conducted by one author (Y. Chida) and verified by another (M. Hamer). Quality and validity were assessed independently by at least two reviewers. Disputes were settled by consensus.

Data synthesis and analysis Meta-analytic procedures were followed that have been described elsewhere [20, 21]. Briefly, an effect size was calculated from the difference between the diabetes and control groups or the incidence between the control and exposed groups. In the case of association between adverse psychosocial factors and diabetes control, this was then transformed into $r$, which gives more weight to larger studies, since they tend to obtain more reliable estimates of the population effect size. If the raw data were not presented, then an $F$ ratio for the main effect over time was used for conversion into $r$ instead. If no relevant convertible statistics were presented, other than a $p$ value, we calculated the $t$ statistic from the
Fig. 1 Flow diagram of systematic review (The Quality of Reporting of Meta-analyses [QUOROM] statement flow diagram)

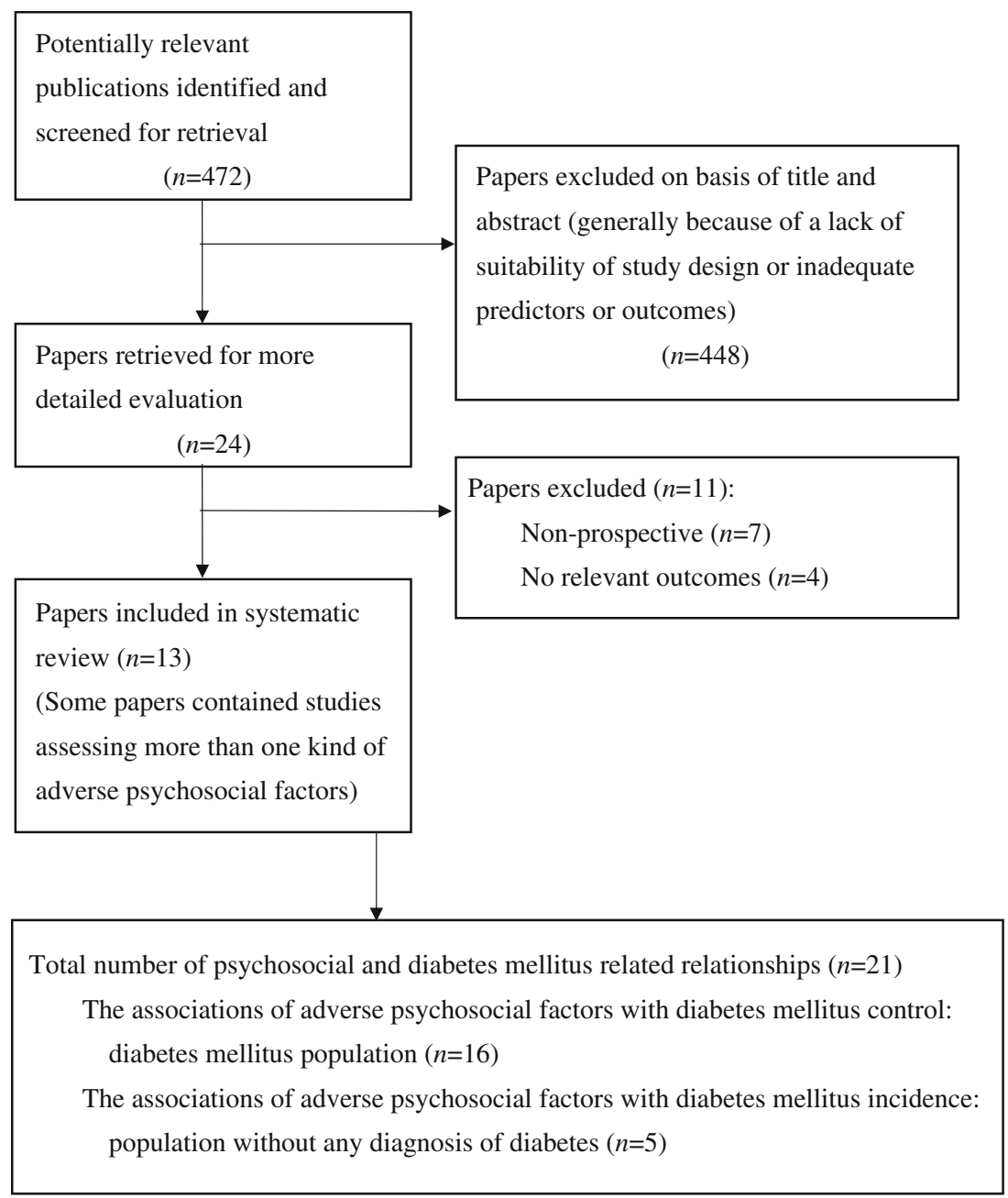




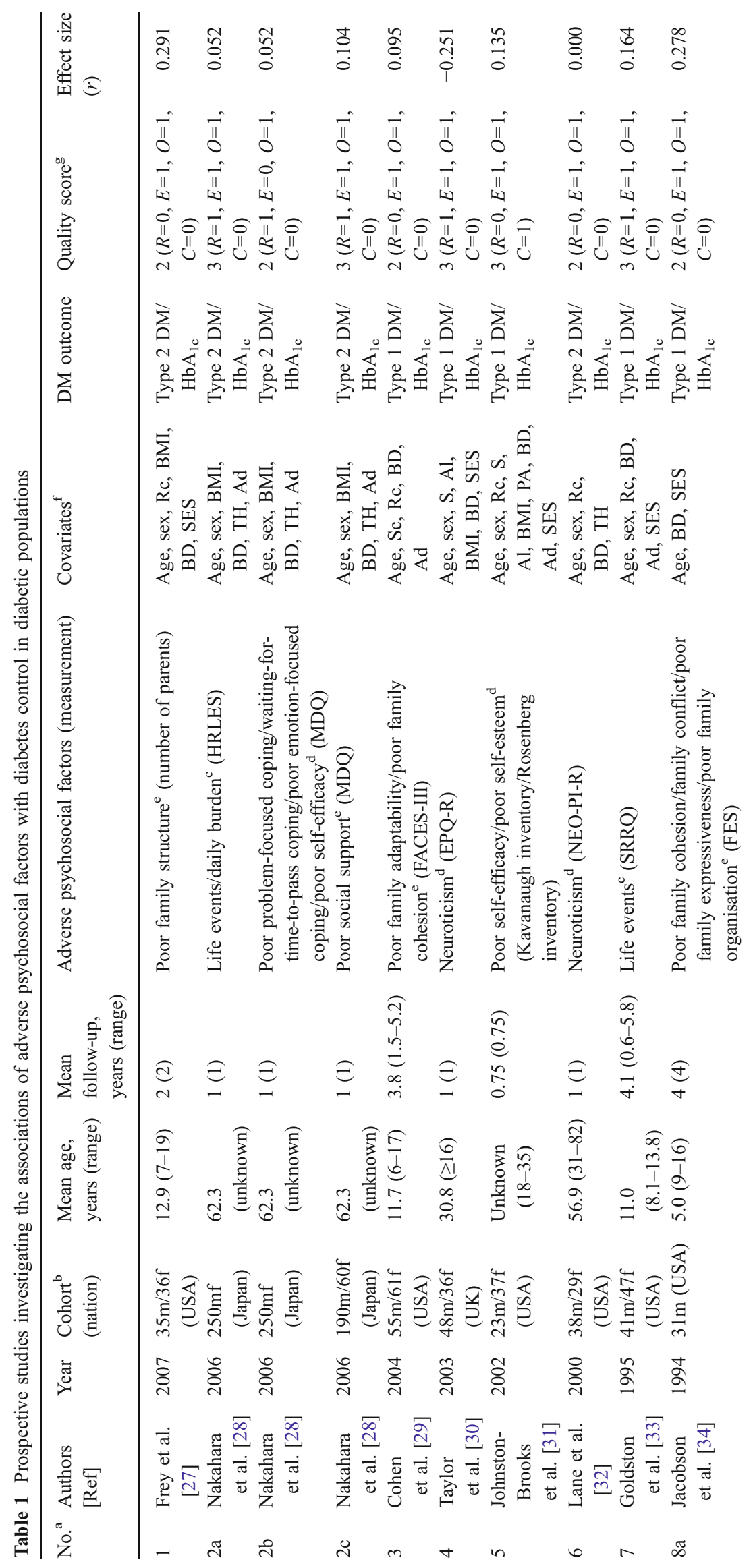




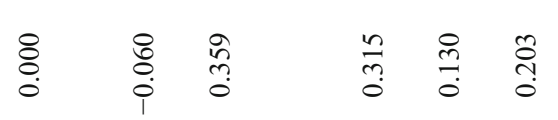

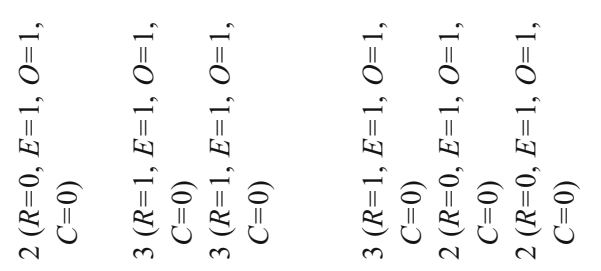

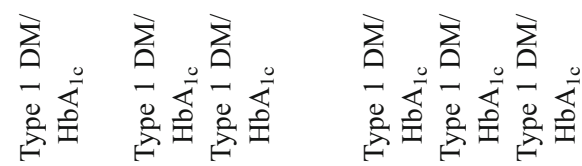

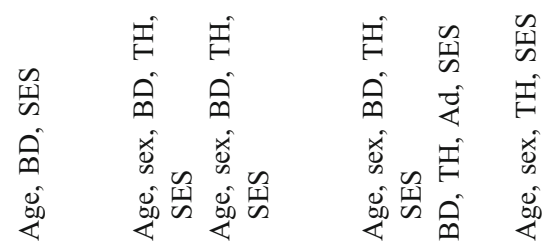

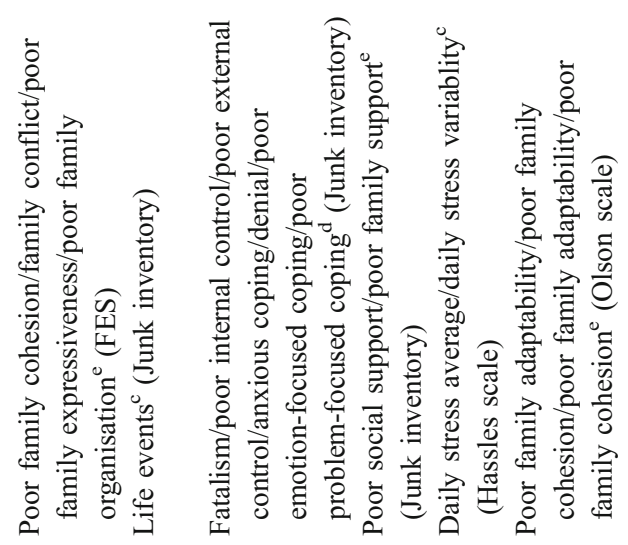

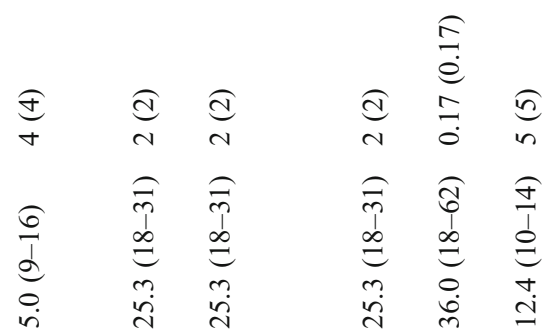

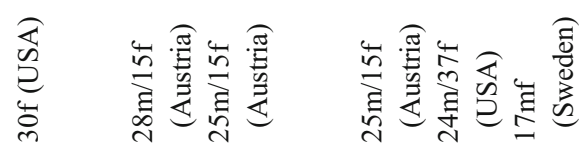

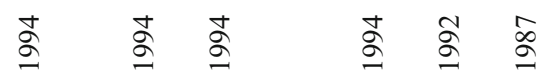

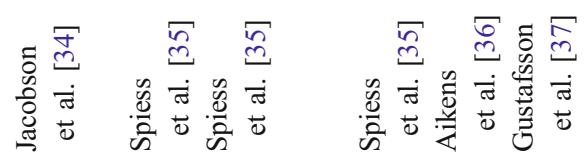

$\infty$ ๙ ค ஃ $๐=$

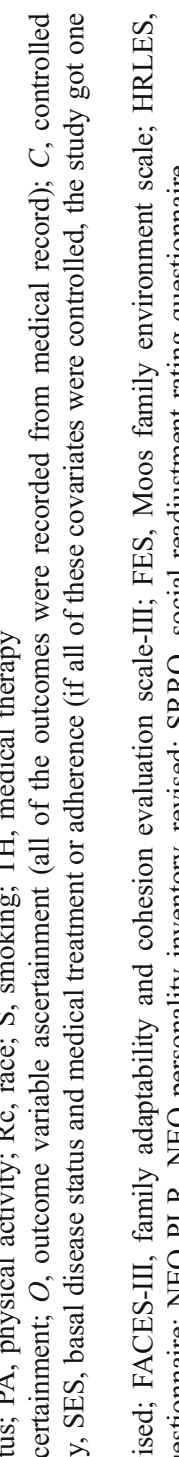

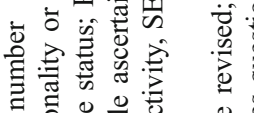

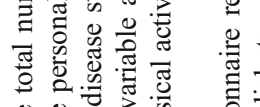

o

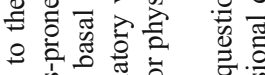

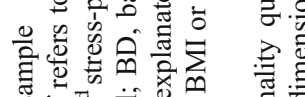

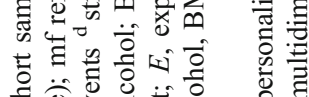

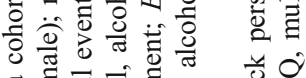

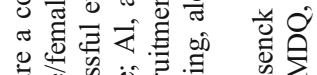

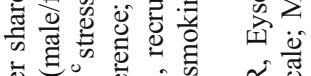

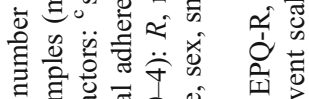

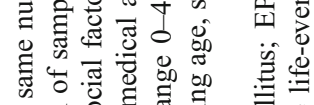

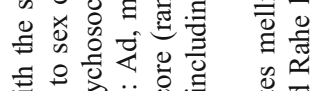

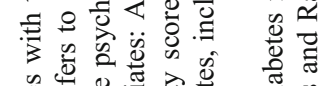

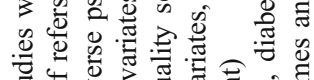

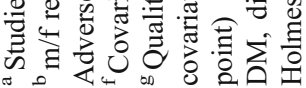




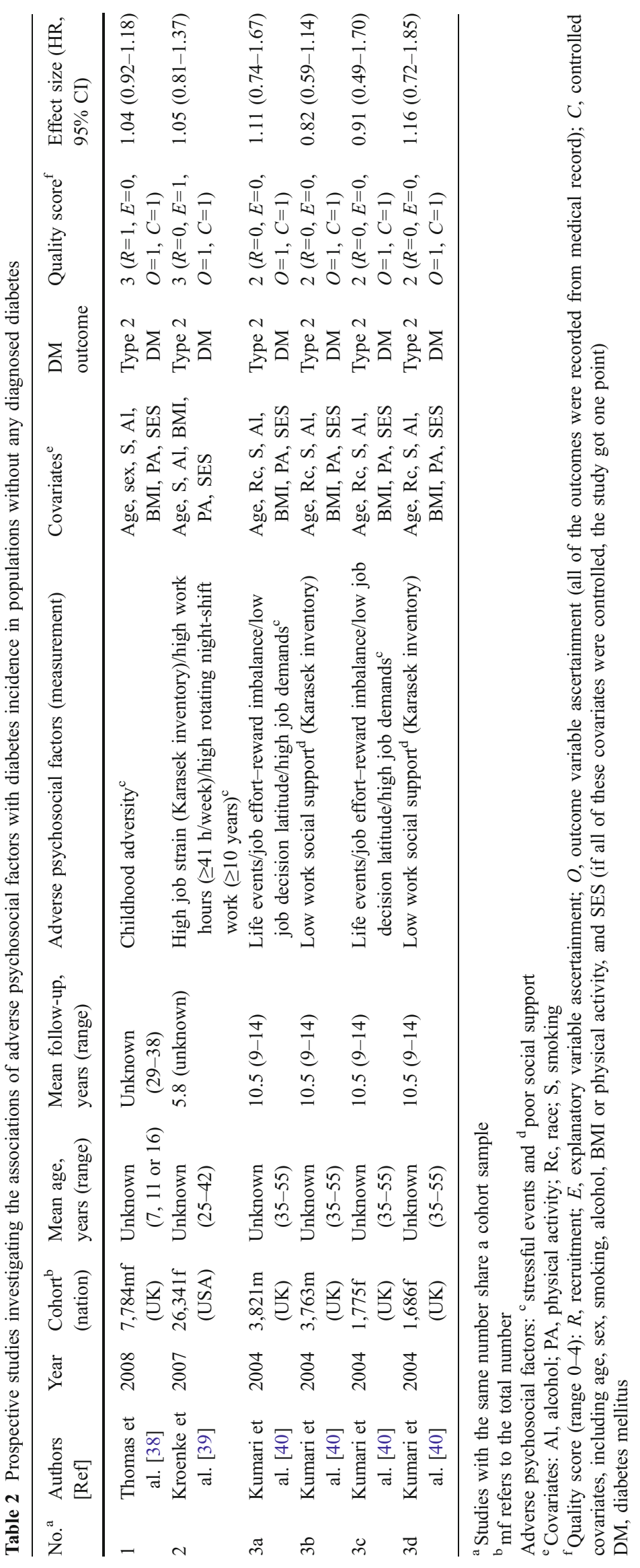


$p$ value and an $r$-sub (equivalent) [22]. When a paper reported $p<0.05, p<0.10$ or NS, we computed $r$-sub (equivalent) with $p$ values of $0.025,0.050,0.50$ (one-tailed), respectively, which likely yielded a highly conservative estimate of the effect size. We performed random effects modelling [23] in our analyses, which takes into account the amount of variance caused by differences between studies as well as differences among participants within studies. Therefore, an inferential statistic or effect size was used to calculate a $z$ score for each study, which was weighted by sample size and then modified by the estimated between-study variance. In the case of association between adverse psychosocial factors and incident diabetes, HRs or RRs were calculated as measures of effect size. In each case, HRs or RRs were logarithmically $\left(\log _{\mathrm{e}}, \mathrm{ln}\right)$ transformed, and standard errors and corresponding $95 \%$ CIs were calculated from $\ln (\mathrm{HR})$ or $\ln (\mathrm{RR})$. Meta-analyses normally include only one effect size per construct per study. In the present meta-analysis, the concept of construct was defined such that each psychosocial category measure was viewed as a separate construct. Thus, several effect sizes were often derived from a given study, each representing a different construct. When more than one measure was reported for a particular construct (e.g. several measures of stressful events), the unweighted mean of each set of effect sizes (e.g. mean $r$ for all stressful events) was used to calculate the effect size. Separate meta-analyses were carried out for each of the three categories of psychosocial factor for the diabetes mellitus control and incidence. If more than one psychosocial factor was analysed in a single article, they were included as separate studies. Provided there was sufficient information (three or more studies), we aimed to perform sensitivity analyses according to the characteristics of the study population (age and type 1 or type 2 diabetes), methodological study quality and type of adverse psychosocial factors. We simultaneously employed the $I^{2}$ statistic for homogeneity between studies, which indicates the proportion of the total variation across studies that is not explained by chance [24]. Finally, to detect publication biases, we measured the degree of asymmetry by using Egger's unweighted regression asymmetry test [25]. All analyses were performed using the Meta-Analysis Program [26].

\section{Results}

A flow diagram detailing the number of studies selected for the present systematic review is shown in Fig. 1. Tables 1 and 2, and the Electronic supplementary material (ESM Table 1), detail the papers that were included in $(n=14)$ [27-40] and excluded from $(n=11)$ the review. There were 16 relationships between psychosocial measures and disease-related factors in populations with diabetes and six relationships between psychosocial measures and diseaserelated factors in populations without diabetes at baseline.

Study characteristics and quality Results from 11 diabetes cohorts and three cohorts without any diagnosed diabetes have been published over the two decades between 1987 and 2008, involving participants from a wide range of countries in Asia, Australasia, Europe and America. The studies of populations without any diagnosed diabetes involved larger samples and a higher proportion of these studies had longer follow-up periods compared with the diabetes studies (sample size \pm SD: 7,528.3 $\pm 9,477.7$ vs $94.0 \pm 81.2$, respectively; $\geq 2$ years follow-up: $100 \%$ vs $50 \%$,

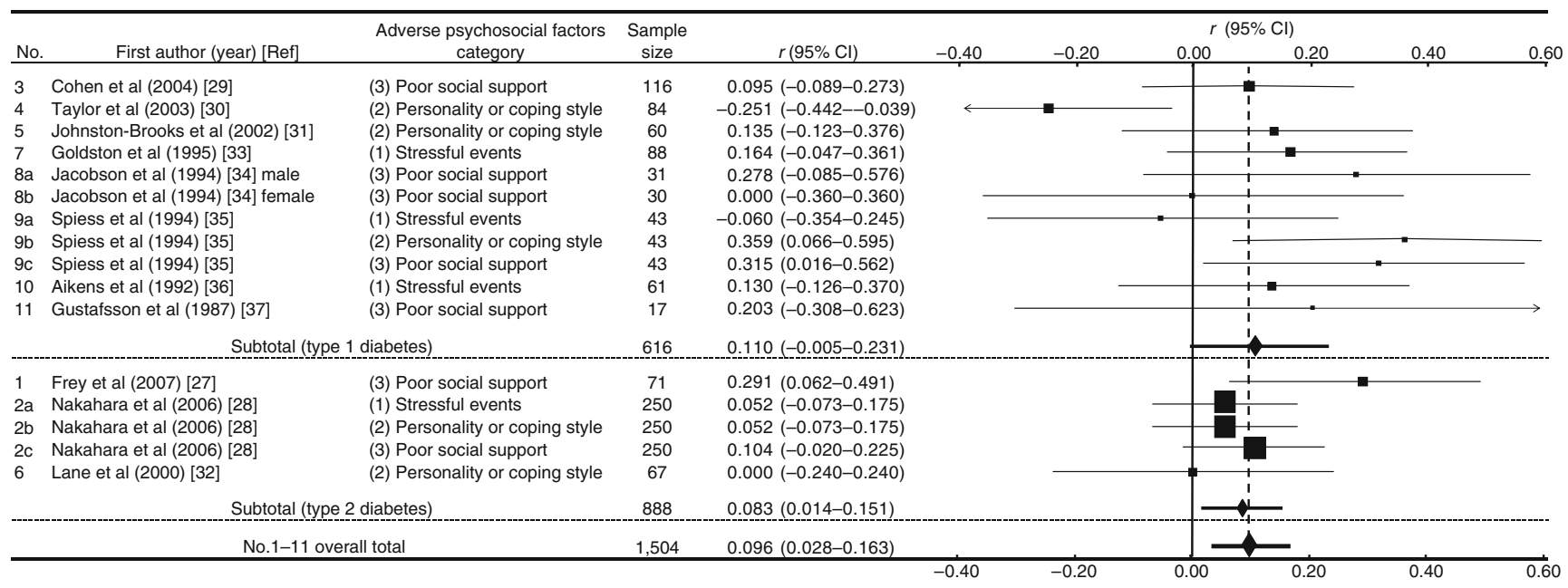

Fig. 2 Forest plots of individual studies investigating the association between adverse psychological factors and diabetes mellitus. Individual study symbols are proportional in size to the weight of the study.
Only those studies for which effect sizes could be computed have been included 


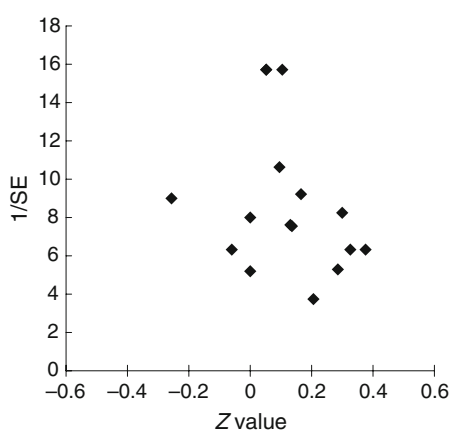

Fig. 3 Funnel plots depicting the relationship between adverse psychological factors and diabetes mellitus. Overall association between adverse psychological factors and diabetes in diabetic populations

respectively). Stressful events were principally evaluated in the studies of populations without any diagnosed diabetes. By contrast, other categories of adverse psychosocial factors were assessed more frequently in diabetes studies than in populations without any diagnosed diabetes. Regarding the type of diabetes, diabetes mellitus population studies estimated type 1 more frequently than type 2 diabetes, while the population without any diagnosed diabetes included only type 2 diabetes. The study quality score $(0-4)$ of the diabetes and non-diabetes studies averaged $2.5 \pm 0.5$ and $2.3 \pm 0.5$, respectively.

Study results and meta-analysis The overall combined $r$ value was $0.096(95 \%$ CI $0.028-0.163, p=0.006)$ for the diabetes studies (Fig. 2) and the overall combined HR was $1.03(95 \%$ CI $0.93-1.13, p=0.61)$ for the populations without any diagnosed diabetes, suggesting a harmful effect of adverse psychosocial factors on diabetes control in the diabetic population. Notably, the overall finding in diabetic populations was not accompanied by publication bias (see Fig. 3). The total variation across studies was $25 \%$ (95\% CI 26-61\%).

As shown in Fig. 4, the subgroup meta-analyses on diabetes studies with longer follow-up periods ( $\geq 2$ years) and younger people ( $\leq 18$ years old) showed significant combined $r$ values $(0.217$, CI $0.093-0.3334$; and 0.129 , CI 0.007-0.248, respectively). However, the diabetes studies with higher quality scores $(\geq 3)$ or larger sample sizes $(\geq 70)$ demonstrated slightly reduced combined $r$ values compared with the overall effect size. Unfortunately, there were insufficient studies to carry out further subanalyses for populations without any diagnosed diabetes.

Differences were observed between the adverse psychosocial factor categories. In the diabetes studies, poor social support was more strongly associated with a poorer outcome compared with the overall effect $(0.149$, CI 0.066-0.231). In contrast, the associations between stressful events and diabetes and between stress-prone personality or coping style and diabetes mellitus were no longer significant. The analyses of diabetes type demonstrated that, when analysed together, type 1 and type 2 diabetic populations exhibited similar associations with adverse psychosocial factors, while type 1 diabetes alone did not show significant associations with adverse psychosocial factors.

\section{Discussion}

The present investigation is the first quantitative systematic review to show that adverse psychosocial factors are significantly associated with poorer control of type 2 diabetes and tend to exacerbate type 1 diabetes in participants with existing diabetes. There was no association between adverse psychosocial factors and diabetes in cohorts without diabetes at baseline, although this finding should be interpreted with caution owing to limited studies. Intriguingly, poor social support was more strongly associated with poor diabetes mellitus control rather than stressful events per se and stress-prone personality or coping style.

Possible underlying mechanisms The harmful relationship between adverse psychosocial factors and subsequent diabetes control might be primarily mediated via behavioural pathways, although we were unable to carry out a

\begin{tabular}{|c|c|c|c|c|c|c|c|c|c|c|c|}
\hline \multirow[b]{2}{*}{ Type of analysis } & \multirow{2}{*}{\multicolumn{2}{|c|}{$\begin{array}{c}\text { No. of } \\
\text { studies (\%) }\end{array}$}} & \multicolumn{2}{|c|}{ Combined effect size } & \multirow{2}{*}{$\begin{array}{c}\text { Publication bias } \\
\quad p \text { value }\end{array}$} & \multirow{2}{*}{$\begin{array}{c}\text { Heterogeneity } \\
I^{2}(95 \% \mathrm{Cl}) \\
\end{array}$} & \multicolumn{5}{|c|}{$r(95 \% \mathrm{Cl})$} \\
\hline & & & $r(95 \% \mathrm{Cl})$ & $p$ value & & & -0.10 & 0.00 & 0.10 & 0.20 & 0.30 \\
\hline Overall analysis & 16 & $(100)$ & $0.096(0.028-0.163)$ & 0.006 & 0.31 & $0.25(0.26-0.61)$ & & & & & \\
\hline Sample size $\geq 70$ & 7 & $(43.8)$ & $0.071(-0.023-0.163)$ & 0.14 & 0.36 & $0.41(0.32-0.43)$ & & & & & \\
\hline Follow-up $\geq 2$ years & 7 & (43.8) & $0.217(0.093-0.334)$ & $<0.001$ & 0.57 & $0.00(0.00-0.23)$ & & & & & \\
\hline Young population $\leq 18$ years old & 4 & (25.0) & $0.129(0.007-0.248)$ & 0.039 & 0.84 & $0.00(0.00-0.00)$ & & & & & \\
\hline Study quality score $\geq 3$ & 8 & $(50.0)$ & $0.088(-0.028-0.202)$ & 0.14 & 0.65 & $0.47(0.49-0.67)$ & & & & & \\
\hline Stressful events & 4 & (25.0) & $0.074(-0.020-0.168)$ & 0.12 & 0.94 & $0.00(0.00-0.00)$ & & & & & \\
\hline Stress-prone personality or coping style & 5 & (31.3) & $0.043(-0.128-0.212)$ & 0.62 & 0.76 & $0.51(0.33-0.54)$ & & & & & \\
\hline Poor social support & 7 & $(43.8)$ & $0.149(0.066-0.231)$ & $<0.001$ & 0.30 & $0.00(0.00-0.00)$ & & & & & \\
\hline Type 1 diabetes & 11 & $(68.8)$ & $0.110(-0.005-0.220)$ & 0.060 & 0.35 & $0.34(0.36-0.64)$ & & & & & \\
\hline Type 2 diabetes & 5 & (31.3) & $0.083(0.014-0.151)$ & 0.018 & 0.49 & $0.00(0.00-0.00)$ & & & & & \\
\hline
\end{tabular}

Fig. 4 Results of meta-analyses, subgrouping, and sensitivity analyses. Higher positive correlation coefficients indicate a more detrimental role of stress in diabetes control 
subgroup analysis because only two studies adjusted for major behavioural covariates (smoking, alcohol consumption, BMI or physical activity level and SES). For example, adverse psychosocial factors might promote high-risk behaviours, such as poor diet, less physical activity, smoking, excessive alcohol consumption, poor sleep and lower treatment adherence [10]. Another possible explanation is that direct physiological pathways are involved in associations between adverse psychosocial factors and poor diabetes control. Adverse psychosocial factors stimulate the hypothalamuspituitary-adrenal axis and the sympathetic nervous system, resulting in increased release of cortisol, adrenaline (epinephrine) and neuropeptide Y. Cortisol is a stress hormone that triggers glucose production, increases lipolysis and circulating NEFA, decreases insulin secretion from beta cells and decreases sensitivity to insulin [41, 42]. Adrenaline has similar effects to cortisol on glucose and fat metabolism [41, 42], and neuropeptide $\mathrm{Y}$ may mediate stress-induced obesity and the metabolic syndrome by increasing adipogenesis and lipolysis [43], thereby resulting in poor control of diabetes.

Issues arising from the sensitivity analyses The subgroup analyses showed slightly reduced effects in studies with larger sample sizes compared with the overall effect, although those with longer follow-up periods exhibited stronger effects. Generally, the cohort studies with larger sample sizes and longer follow-up periods are considered to provide stronger evidence, because these designs increase the power to detect any differences between the control and exposed groups. Furthermore, study quality is important [44], and the subgroup analysis of studies with a high quality score $(\geq 3)$ showed a slightly reduced association between adverse psychosocial factors and poor diabetes control compared with the overall association. Nevertheless, in the present meta-analyses, we found no evidence of publication bias - a positive result bias is obtained if authors are more likely to submit, or editors accept, positive than null (negative or inconclusive) results.

The subgroup meta-analyses across the three different types of adverse psychosocial factors found that low social support was more strongly associated with poorer diabetes control than were stressful events and stress-prone personality or coping style. Given that the disease burden of diabetes can in itself be considered a powerful chronic stressor, this may explain why factors such as social support are important predictors of diabetes control. Randomised controlled trials have shown that psychological interventions have an efficient effect on glycaemic control in patients with type 1 or type 2 diabetes [4, 5], which suggests that organising social support may be rather more important than preventing stressful events in relation to diabetes or managing human cognition, appraisal and coping. It is also interesting that adverse psychosocial factors predicted diabetes control in younger populations ( $\leq 18$ years old), suggesting that young individuals are more likely to respond to adverse psychosocial factors. In line with this finding, the meta-analysis conducted by Winkley et al. [5] showed that psychological interventions are effective in children and adolescents, but not adults. More intriguingly, the analyses of diabetes types found that adverse psychosocial factors were more robustly associated with poor control in type 2 than type 1 diabetes, although possible explanations of this difference remain to be identified.

Limitations and guidelines for future studies The aetiological association of adverse psychosocial factors with diabetes remains undetermined at present because only two cohorts have been published. However, a recent metaanalysis of nine prospective studies reported that depressed adults have a $37 \%$ increased risk of developing type 2 diabetes [45]. Negative life events during the first 2 years of life, such as divorce, high parenting stress and foreign origin of the mother predicted an increased risk of diabetesrelated autoimmunity, which critically contributes to the development of type 1 diabetes [46, 47]. In addition, inflammatory cytokines such as IL-6 are known to increase in response to acute psychosocial stress [48], and these inflammatory markers have also been prospectively associated with an increased risk of diabetes among a large cohort of postmenopausal women [49]. Taken together, these findings indicate that a relationship between adverse psychosocial factors and the development of diabetes is feasible.

Additional prospective research is needed to examine associations between psychosocial factors and diabetes risk in populations without any diagnosed diabetes. Studies should endeavour to control for all putative behavioural and socioeconomic covariates, such smoking, drinking, sleep disturbance, physical activity and treatment adherence. The measurement of possible psychobiological markers that might mediate the association between adverse psychosocial factors and risk of diabetes, such as neuroendocrine function (e.g. cortisol, catecholamines and neuropeptides) and immunological processes (diabetes-related autoantibodies) would also advance this field.

\section{Conclusion}

The current findings on the association of adverse psychosocial factors with poor diabetes prognosis point to the value of further research in the field, but they should be interpreted with caution and additional prospective research is needed. 
Acknowledgements We are grateful to colleagues in many research centres for providing the additional data required for meta-analysis. This study was supported by the Kanae Foundation for the Promotion of Medical Science and the British Heart Foundation.

Duality of interest The authors declare that there is no duality of interest associated with this manuscript.

\section{References}

1. Allan FN (1953) The writings of Thomas Willis, M.D. Diabetes three hundred years ago. Diabetes 2:74-77

2. Brotman DJ, Golden SH, Wittstein IS (2007) The cardiovascular toll of stress. Lancet 370:1089-1100

3. McEwen BS (1998) Protective and damaging effects of stress mediators. N Engl J Med 338:171-179

4. Ismail K, Winkley K, Rabe-Hesketh S (2004) Systematic review and meta-analysis of randomized controlled trials of psychological interventions to improve glycaemic control in patients with type 2 diabetes. Lancet 363:1589-1597

5. Winkley K, Landau S, Eisler I, Ismail K (2006) Psychological interventions to improve glycaemic control in patients with type 1 diabetes: systematic review and meta-analysis of randomised controlled trials. BMJ 333:65

6. Surwit RS, Schneider MS, Feinglos MN (1993) Stress and diabetes mellitus. Diabetes Care 15:1413-1422

7. Cosgrove M (2004) Do stressful life events cause type 1 diabetes? Occup Med (Lond) 54:250-254

8. Peng H, Hagopian W (2006) Environmental factors in the development of type 1 diabetes. Rev Endocr Metab Disord $7: 149-162$

9. Kramer JR, Ledolter J, Manos GN, Bayless ML (2000) Stress and metabolic control in diabetes mellitus: methodological issues and an illustrative analysis. Ann Behav Med 22:17-28

10. Lloyd C, Smith J, Weinger K (2005) Stress and diabetes: a review of the links. Diabetes Spectrum 18:121-127

11. Brosschot JF, Gerin W, Thayer JF (2006) The preservative cognition hypothesis: a review of worry, prolonged stress-related physiological activation, and health. J Psychosom Res 60:113-124

12. Graham JE, Christian LM, Kiecolt-Glaser JK (2007) Close relationships and immunity. In: Ader R (ed) Psychoneuroimmunology. 4th edn. Academic Press, Amsterdam, pp 781-798

13. Stroup DF, Berlin JA, Morton SC et al (2000) Meta-analysis of observational studies in epidemiology. JAMA 283:2008-2012

14. Pcikering T (1999) Cardiovascular pathways: socioeconomic status and stress effects on hypertension and cardiovascular function. Ann NY Acad Sci 896:262-277

15. Wright RJ, Subramanian SV (2007) Advancing a multilevel framework for epidemiologic research on asthma disparities. Chest 132:S757-S769

16. McEwen BS (2007) Physiology and neurobiology of stress and adaptation: central role of the brain. Physiol Rev 87:873-904

17. Laupacis A, Wells G, Richardson S, Tugwell P (1994) Users' guides to the medical literature. V. How to use an article about prognosis. JAMA 272:234-237

18. Chida Y, Steptoe A (2008) Positive psychological well-being and mortality: a quantitative review of prospective observational studies. Psychosom Med 70:741-756

19. Chida Y, Steptoe A, Powel LH (2008) Religiosity/spirituality and mortality: A systematic quantitative review. Psychother Psychosom (in press)
20. Chida Y, Hamer M, Steptoe A (2008) A bidirectional relationship between psychosocial factors and atopic disorders: a systematic review and meta-analysis. Psychosom Med 70:102-116

21. Chida Y, Hamer H (2008) Chronic psychosocial factors and acute physiological responses to laboratory induced stress in the healthy populations: A quantitative review of 30 years of investigations. Psychol Bull (in press)

22. Rosenthal R, Rubin DB (2003) $r_{\text {equivalent }}$ a simple effect size indicator. Psychol Methods 8:492-496

23. DerSimonian R, Laird N (1986) Meta-analysis in clinical-trials. Control Clin Trials 7:177-188

24. Egger M, Smith GD, Schneider M, Minder C (1997) Bias in meta-analysis detected by a simple, graphical test. BMJ 315: 629-634

25. Masui K (2003) Meta-analysis software user's guide (written in Japanese). In: Masui K (ed) Kokokara-hajimeru meta-analysis. Shinko-Koueki Medical Press, Tokyo, pp 91-121

26. Ioannidis J, Patsopoulos N, Evangelou E (2007) Uncertainty in heterogeneity estimates in meta-analyses. BMJ 335:914-916

27. Frey MA, Templin T, Ellis D, Gutai J, Podolski C-L (2007) Predicting metabolic control in the first $5 \mathrm{yr}$ after diagnosis for youths with type 1 diabetes: the role of ethnicity and family structure. Pediatr Diabetes 8:220-227

28. Nakahara R, Yoshiuchi K, Kumano H, Hara Y, Suematsu H, Kuboki T (2006) Prospective study on influence of psychosocial factors on glycemic control in Japanese patients with type 2 diabetes. Psychosomatics 47:240-246

29. Cohen DM, Lumley MA, Naar-King S, Partridge T, Cakan N (2004) Child behavior problems and family functioning as predictors of adherence and glycemic control in economically disadvantaged children with type 1 diabetes: a prospective study. J Pediatr Psychol 29:171-184

30. Taylor MD, Frier BM, Gold AE, Deary IJ (2003) Psychosocial factors and diabetes-related outcomes following diagnosis of type 1 diabetes in adults: the Edinburgh prospective diabetes study. Diabetes Med 20:135-146

31. Johnston-Brooks CH, Lewis MA, Garg S (2002) Self-efficacy impacts self-care and $\mathrm{HbA}_{1 \mathrm{c}}$ in young adults with type 1 diabetes. Psychosom Med 64:43-51

32. Lane JD, Parekh PI, McCaskill CC, Feinglos MN, Williams PG, Surwit RS (2000) Personality correlates of glycemic control in type 2 diabetes. Diabetes Care 23:1321-1325

33. Goldston DB, Kovacs M, Obrosky DS, Iyengar S (1995) A longitudinal study of life events and metabolic control among youths with insulin-dependent diabetes mellitus. Health Psychol 14:409-414

34. Jacobson AM, Hauser ST, Lavori P et al (1994) Family environment and glycemic control: A four-year prospective study of children and adolescents with insulin-dependent diabetes mellitus. Psychosom Med 56:401-409

35. Spiess K, Sachs G, Moser G, Pietschmann P, Schernthaner G, Prager R (1994) Psychological moderator variables and metabolic control in recent onset type 1 diabetic patients - a two year longitudinal study. J Psychosom Res 38:249-258

36. Aikens JE, Wallander JL, Bell DSH, Cole JA (1992) Daily stress variability, learned resourcefulness, regimen adherence, and metabolic control in type 1 diabetes mellitus: evaluation of a path model. J Consult Clin Psychol 60:113-118

37. Gustafsson PA, Cederblad M, Ludvigsson J, Lundin B (1987) Family interaction and metabolic balance in juvenile diabetes mellitus. A prospective study. Diabetes Res Clin Pract 4:7-14

38. Thomas C, Hyppönen E, Power C (2008) Obesity and type 2 diabetes risk in midadult life: The role of childhood adversity. Pediatrics 121:e1240-e1249 
39. Kroenke CH, Spiegelman D, Manson J, Schernhammer ES, Colditz GA, Kawachi I (2007) Work characteristics and incidence of type 2 diabetes in women. Am J Epidemiol 165:175-183

40. Kumari M, Head J, Marmot M (2004) Prospective study of social and other risk factors for incidence of type 2 diabetes in the Whitehall II study. Arch Intern Med 164:1873-1880

41. Lundberg U (2005) Stress hormones in health and illness: the role of work and gender. Psychoneuroendocrinology 30:1017-1021

42. Bhathena SJ (2006) Relationship between fatty acids and the endocrine and neuroendocrine system. Nutr Neurosci 9:1-10

43. Kuo LE, Kitlinska JB, Tilan JU et al (2006) Neuropeptide Y acts directly in the periphery on fat tissue and mediates stress-induced obesity and metabolic syndrome. Nature Med 13:803-811

44. LeLorier J, Gregoire G, Benhaddad A, Lapierre J, Derderian F (1997) Discrepancies between meta-analyses and subsequent large randomized, controlled trials. N Engl J Med 337:536-542
45. Knol MJ, Twisk JWR, Beekman ATF, Heine RJ, Snoek FJ, Pouwer F (2006) Depression as a risk factor for the onset of type 2 diabetes mellitus. A meta-analysis. Diabetologia 49:837-845

46. Sepa A, Frodi A, Ludvigsson J (2005) Mothers' experiences of serious life events increase the risk of diabetes-related autoimmunity in their children. Diabetes Care 28:2394-2399

47. Sepa A, Wahlberg J, Vaarala O, Frodi A, Ludvigsson J (2005) Psychological stress may induce diabetes-related autoimmunity in infancy. Diabetes Care 28:290-295

48. Steptoe A, Hamer M, Chida Y (2007) The effects of acute psychological stress on circulating inflammatory factors in humans: a review and meta-analysis. Brain Behav Immun 21:901-912

49. Liu S, Tinker L, Song YQ et al (2007) A prospective study of inflammatory cytokines and diabetes mellitus in a mutli-ethnic cohort of postmenopausal women. Arch Intern Med 167:16761685 